\title{
The origin of CDK regulation
}

\author{
Anatoliy Li and J. Julian Blow \\ CRC Chromosome Replication Research Group, Wellcome Trust Biocentre, University of \\ Dundee, Dow Street, Dundee DD1 5EH, UK
}

\section{Abstract}

The initiation of DNA replication is restrained by Cip/Kip proteins that inhibit Cdk2. Degradation of Xenopus Xic1, a Kip1 orthologue, is dependent on its recruitment to replication origins. This ensures that activation of $\mathrm{Cdk} 2$ and (subsequent initiation of replication) is co-ordinately regulated at, and localised to, replication origins.

Cell cycle progression in eukaryotic cells, including the decision to initiate DNA replication, is driven by cyclin-dependent kinases (CDKs). The catalytic subunit of CDK is only active as a protein kinase when bound to a regulatory cyclin protein, and CDK activity can be inhibited by binding CDK inhibitors (CKIs). While the level of CDKs does not change significantly during the cell cycle, the abundance of cyclins and CKIs are tightly controlled, both temporally and spatially, providing precise regulation of cell cycle events. Although the cell cycle regulation of CDKs has been extensively characterized, only a few physiological CDK substrates have been identified, and the detailed mechanisms by which CDKs regulate cell-cycle transitions are still unclear. The paper by Furstenthal and co-authors on page xxx of this issue ${ }^{1}$ reveals a new pathway by which CKI abundance is regulated by CDKs in Xenopus (frog) eggs. These new results show that Xic1, a Xenopus CKI, is only degraded when bound to cyclin E/Cdk2 that is associated with replication origin proteins on the DNA. This provides further insights into how events occuring at replication origins can regulate the progression of cells into $\mathrm{S}$ phase of the cell cycle.

The Cip/Kip family of CKIs, found in most higher eukaryotes, binds and inhibits the activity of cyclin $\mathrm{E} / \mathrm{Cdk} 2$ and cyclin $\mathrm{A} / \mathrm{Cdk} 2$, both involved in DNA replication ${ }^{2,3}$. Any inhibitory activity provided by Cip/Kip proteins must therefore be abolished before DNA replication can occur. This is in part achieved by proteolysis of Cip/Kip by the proteasome at the G1/S phase transition. In Xenopus eggs two closely-related CKIs have been identified, called Xic1 and Kix1, that are orthologues of mammalian Kip ${ }^{4,5}$. Like other Kip proteins, Xic1 is degraded following ubiquitylation by the SCF ubiquitin ligase ${ }^{6-8}$. In human cells, targeting of Kip1 proteins for ubiquitylation by the ubiquitin ligase SCF requires its prior phosphorylation by $\mathrm{CDK}^{9-11}$.

Furstenthal et al now reveal a new pathway regulating Xic1 ubiquitylation and degradation in Xenopus, where in contrast with the current paradigm, direct phosphorylation of Xic1 by cyclin $\mathrm{E} / \mathrm{Cdk} 2$ is not essential for its destruction. Nevertheless, the presence of active cyclin $\mathrm{E} / \mathrm{Cdk} 2$ kinase is still necessary for Xic1 ubiquitylation. The authors have solved this conundrum by showing that the recruitment of Xic1 to chromatin is dependent on the physical presence of cyclin E/Cdk2 (with or without kinase activity). Both recruitment of Xic1 to chromatin and activation of the ubiquitylation machinery by cyclin E/Cdk2 are required for Xic1 destruction (Fig 1, left). The recruitment of Xic1 to chromatin also depends on the presence of two other protein bound to replication origins, the origin recognition complex (ORC) and the Cdc6 protein ${ }^{12}$. ORC and Cdc6 (along with another origin protein termed Cdt1 or RLF-B) are also required to load the Mcm2-7 initiation proteins onto chromatin and "license" the origin for replication ${ }^{13}$ (Fig 1, right). To underline 
the importance of this new pathway, components of the SCF ubiquitin ligase are also recruited to chromatin, and this depends on the presence of both cyclin E/Cdk2 and the Mcm2-7 origin proteins. Thus two proteins that play a central role in the initiation of DNA replication - cyclin E/Cdk2 and Mcm2-7 - are co-ordinately regulated at, and localised to, replication origins (Fig 1).

Cyclin E/Cdk2 appears to play at least three roles in driving Xic1 degradation. Firstly, cyclin E/Cdk2 forms a ternary complex with Xic1, and formation of this complex appears necessary for the efficient nuclear import of Xic ${ }^{7}$. This will enhance Xic1 ubiquitylation and degradation since these events only occur in the nucleus. It is currently unclear which domains of Xic1 and cyclin E/Cdk2 are involved in forming the complex. Second, cyclin E/ $\mathrm{Cdk} 2$ is physically required for the recruitment of Xic1 to chromatin, and again kinase activity is dispensable. Instead, chromatin recruitment of Xic1 is mediated by the binding of the cyclin E/Cdk2/Xic1 ternary complex to chromatin. Chromatin recruitment is also dependent on the origin proteins ORC and Cdc6, possibly by an interaction between cyclin E and $\mathrm{Cdc}^{1,12}$ (Fig 1, left).

The third role of cyclin E/Cdk2 in Xic1 degradation is for the recruitment of SCF components to chromatin ${ }^{1}$, which is likely to be required for the efficient ubiquitylation of chromatin-bound Xic1. Recruitment of these SCF components to chromatin and efficient Xic1 ubiquitylation depends on the presence of the Mcm2-7 origin proteins (Fig 1, right). Since the "pre-replicative complex" of ORC, Cdc6, RLF-B/Cdt1 and Mcm2-7 are involved in recruiting both Xic1 (via Cdc6 and cyclin E/Cdk2) and SCF (via Mcm2-7), this provides a mechanism for bringing together SCF and its substrate at origins of replication. Although cyclin $\mathrm{E} / \mathrm{Cdk} 2$ kinase activity is required for ubiquitylation of Xic1, it is currently unclear whether SCF recruitment also requires kinase activity.

In order to understand the functional significance of this regulatory pathway, it is important to remember that cell cycle transitions are abrupt switch-like events, not gradual changes. The regulation of Kip1 ubiquitylation seen in mammalian somatic cells ${ }^{2,3}$ seems to operate as a switch-like positive feedback loop (Fig 2a). During progression through G1 phase, various changes result in the increased availability of cyclin $E$ which is initially restrained by Kip1. Increasing cyclin E/Cdk2 kinase activity then leads to Kip1 phosphorylation, ubiquitylation and degradation. The Xenopus eggs studied by Fursthenthal et $\mathrm{al}^{1}$ face a different problem as they contain large quantities of cyclin E stockpiled for use in the early embryo whose activity must be regulated to provide orderly progression through the cell cycle. It is therefore important that cyclin $\mathrm{E} / \mathrm{Cdk} 2$ is restrained until nuclei have been properly formed in the brief $\mathrm{G} 1$ period $^{13}$. Having Xic1 degradation linked to nuclear import and chromatin recruitment, whilst intranuclear SCF activation is CDK-dependent, also potentially creates a positive feedback loop (Fig $2 b$ ). In this case however, the loop is triggered by chromatin recruitment rather than cyclin availability.

This interpretation is still speculative and other possibilities can be envisaged. One question concerns the relevant substrate for SCF in the early embryonic cell cycle. Xic1 is not thought to be abundant at this stage of development, but levels build up at the mid-blastula transition ${ }^{5}$. So, does the mechanism described by Furstenthal et al only operate at later stages of development, or is there another Xic1-like target for SCF in the early embryo? The latter suggestion is supported by the observation that the Cdc34 component of SCF is essential for DNA replication and cyclin E/Cdk2 activation in the early embryo ${ }^{6}$. Another problem is whether the known behaviour of the Cdc6 origin protein matches the demands made on it by the Xic1 results. Previous work has shown that Cdc6 is found associated with chromatin at two different stages in the early embryonic cell cycle: one peak in late mitosis and early G1 and another peak in mid- to late-S phase ${ }^{13,14}$. However, if recruitment of 
cyclin $\mathrm{E} / \mathrm{Cdk} 2$ to $\mathrm{Cdc} 6$ is necessary for the initiation of replication, Cdc6 would be expected to be seen on chromatin during late G1 and early $\mathrm{S}$ phase. This problem is underlined by the observation that once origin licensing has occurred in the Xenopus system, neither ORC nor Cdc6 are subsequently required for the initiation of DNA replication to occur ${ }^{13}$. We can look forward to further work resolving these apparent discrepancies. Whatever the outcome, the recent work of Furstenthal et $\mathrm{al}^{1}$ provides an important new model to broaden our imagination about how Cip/Kip degradation and CDK activity can potentially be regulated.

\section{References}

1. Furstenthal L, Swanson C, Kaiser BK, Aldridge AG, Jackson PK. Nat. Cell Biol. 2001 in press.

2. Sherr CJ, Roberts JM. Genes Dev. 1999; 13:1501-1512. [PubMed: 10385618]

3. Ekholm SV, Reed SI. Curr. Opin. Cell Biol. 2000; 12:676-684. [PubMed: 11063931]

4. Su JY, Rempel RE, Erikson E, Maller JL. Proc. Natl. Acad. Sci. U. S. A. 1995; 92:10187-10191. [PubMed: 7479751]

5. Shou WY, Dunphy WG. Mol. Biol. Cell. 1996; 7:457-469. [PubMed: 8868473]

6. Yew PR, Kirschner MW. Science. 1997; 277:1672-1676. [PubMed: 9287222]

7. Swanson C, Ross J, Jackson PK. Proc. Natl. Acad. Sci. U. S. A. 2000; 97:7796-7801. [PubMed: 10884410]

8. Yew PR. Chuang LC. J. Biol. Chem. 2001; 276:1610-1617. [PubMed: 11044455]

9. Carrano AC, Eytan E, Hershko A, Pagano M. Nat. Cell Biol. 1999; 1:193-199. [PubMed: 10559916]

10. Montagnoli A, et al. Genes Dev. 1999; 13:1181-1189. [PubMed: 10323868]

11. Tsvetkov LM, Yeh KH, Lee SJ, Sun H, Zhang H. Curr. Biol. 1999; 9:661-664. [PubMed: 10375532]

12. Furstenthal L, Kaiser BK, Swanson C, Jackson PK. J. Cell Biol. 2001; 152:1267-1278. [PubMed: 11257126]

13. Blow JJ. EMBO J. 2001; 20 in press.

14. Jares P, Blow JJ. Genes Dev. 2000; 14:1528-1540. [PubMed: 10859170] 


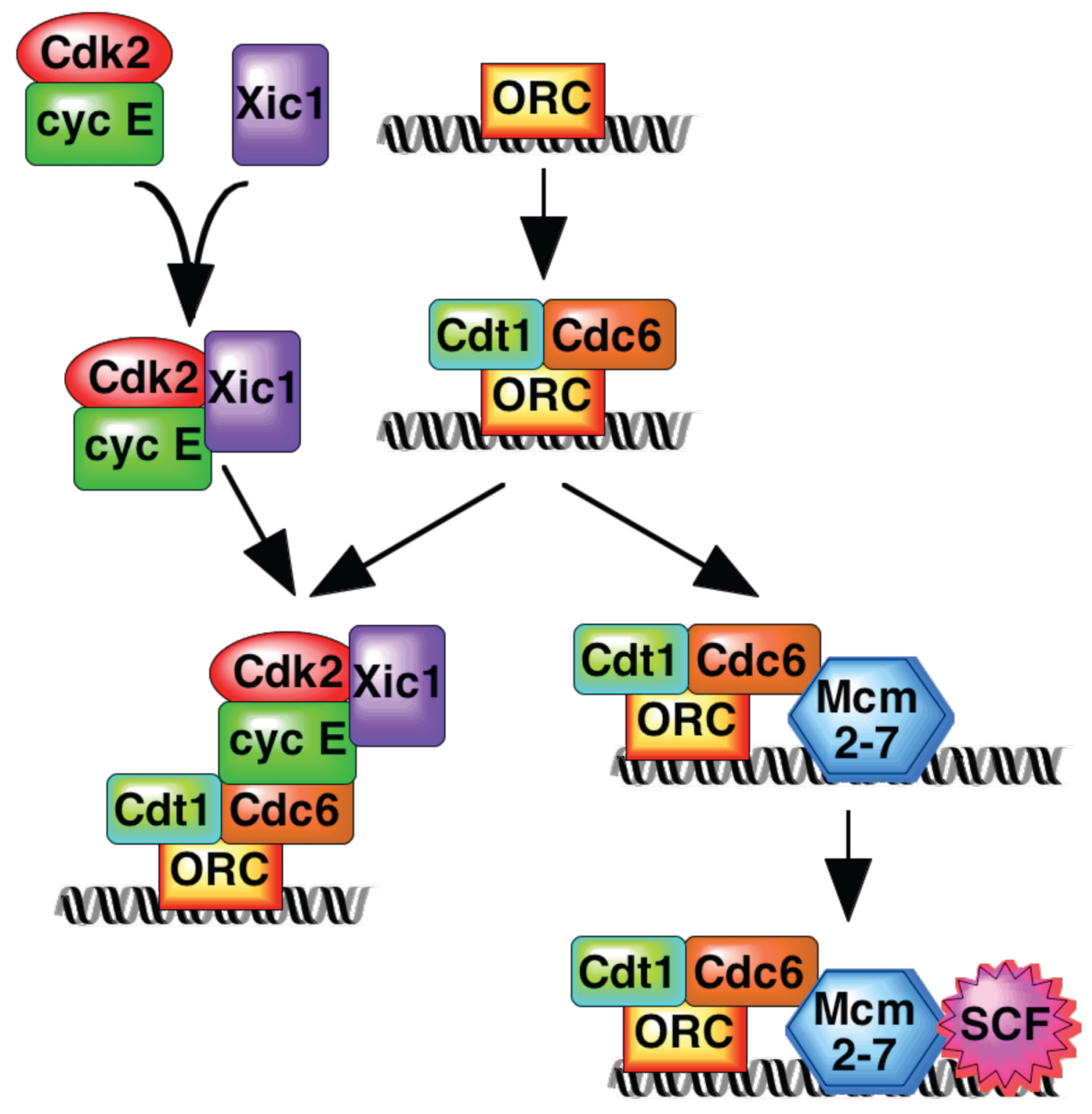

cyclin E/Cdk2 and Xic1 recruitment

\section{Licensing and $\underline{\text { SCF recruitment }}$}

Figure 1.

Recruitment of cyclin E/Cdk2/Xic1 and SCF to replication origins. Xic1 forms a ternary complex with cyclin E/Cdk2 and binds to ORC/Cdc6 at replication origins (left). Mcm2-7 binding to replication origins ("licensing") depends on ORC, Cdc6 and Cdt1; SCF is recruited to licensed chromatin containing Mcm2-7 (right). Although it is likely that both assembly reactions can occur at the same origin, this has not been proven. 

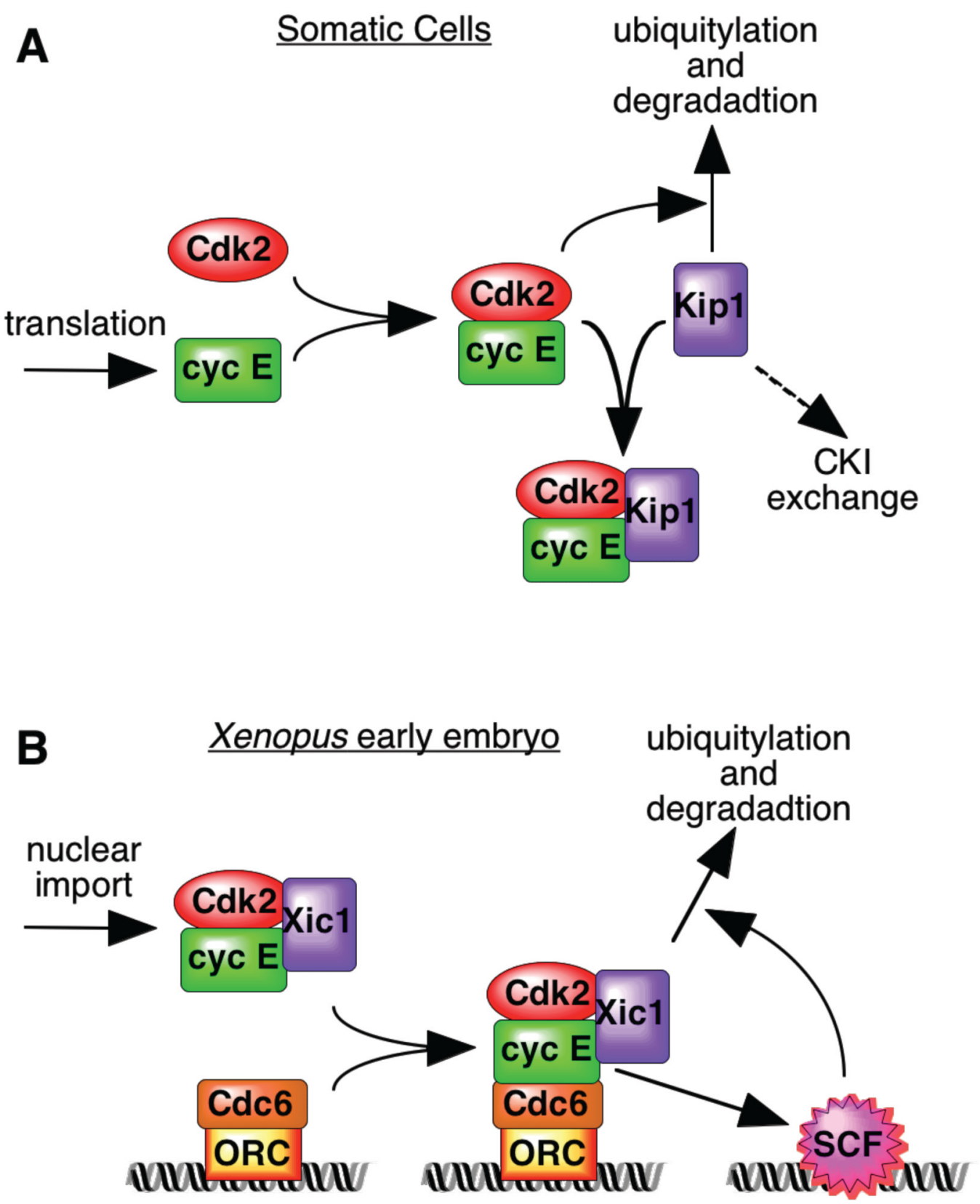

Figure 2.

Different positive feedback loops for regulating Kip degradation in somatic and early embryonic cells. A, In somatic cells, Kip1 ubiquitylation and degradation is dependent on its prior phosphorylation by cyclin $\mathrm{E} / \mathrm{Cdk} 2$. Active cyclin $\mathrm{E} / \mathrm{Cdk} 2$ kinase is provided by a build-up of cyclin E, or by recruitment of Kip1 to cyclin D/Cdk4-6 (CKI exchange ${ }^{3}$ ). B, In the Xenopus early embryo, Xic1 recruitment to chromatin via cyclin $\mathrm{E} / \mathrm{Cdk} 2$ is required for its degradation; cyclin $\mathrm{E} / \mathrm{Cdk} 2$ is also required for localisation of the SCF ubiquitin ligase to chromatin and its activation. 